\title{
Band Engineering in Transition Metal Dichalcogenides: Stacked versus Lateral Heterostructures
}

\author{
Yuzheng Guo, John Robertson
}

Engineering Department, Cambridge University, Cambridge CB2 1PZ, UK

\begin{abstract}
We calculate a large difference in the band alignments for transition metal dichalcogenide (TMD) heterojunctions when arranged in the stacked layer or lateral (in-plane) geometries, using direct supercell calculations. The stacked case follows the unpinned limit of the electron affinity rule, whereas the lateral geometry follows the strongly pinned limit of alignment of charge neutrality levels. TMDs therefore provide one of the few clear tests of band alignment models, whereas three-dimensional semiconductors give less stringent tests because of accidental chemical trends in their properties.
\end{abstract}

The ability to choose semiconductors with different band alignments is important in the design of heterojunction lasers, transistors or multi-junction solar cells [1]. Thus, the band alignments of three-dimensionally bonded semiconductors have been extensively studied. Two-dimensional semiconductors such as transition-metal dichalcogenides (TMDs) have recently attracted attention because, unlike graphene, they possess a band gap. One of their most interesting possible applications is as heterojunctions in tunnel field effect transistors (TFET). TFETs aim to reduce the subthreshold slope of an FET's transfer characteristic below the thermionic limit of 60 $\mathrm{mV} /$ decade $[2,3]$. This requires semiconductor heterojunctions with a narrow type II or type III band gap alignment. TFETs would typically use III-V semiconductors due to their wide range of band gaps and band offsets. However, such three-dimensional semiconductors must be latticematched, otherwise mismatch defects create gap states, increasing the TFET's subthreshold slope. An alternative is to use stacked layer heterostructures of two-dimensional TMDs [4-6]. These require no lattice matching, so incommensurate heterojunctions create no mismatch defects and dangling bonds.

It is therefore important to know the heterojunction band alignments of TMDs, and understand what controls them [7-11]. It is generally assumed that band alignments of stacked layer TMD heterojunctions follow the electron affinity rule, because of the weak van der Waals inter-layer bonding [7-9]. There is now also increasing work on lateral TMD heterojunctions [12-16]. In this case, the two TMDs have direct covalent bonding, as in the three-dimensional case. Heterojunctions could be controlled by the electron affinity rule [17-18], or they could follow an alignment which equalises charge neutrality levels(CNLs) [19-20], in other words the unpinned and strongly pinned limits. It has been quite difficult to determine which limit actually holds in three-dimensional semiconductors, in experiment or in theory, partly because the need for lattice matching in that case gives similar chemical trends for the two limits [21-23]. It turns out that the lateral and stacked TMD heterojunctions provide a useful test of this problem.

We have therefore calculated the ionization potentials (IPs) and electron affinities (EAs) of the TMDs $\left(\mathrm{MX}_{2}, \mathrm{M}=\mathrm{Mo}, \mathrm{W}, \mathrm{Hf}, \mathrm{Zr}, \mathrm{X}=\mathrm{S}\right.$, Se and Te) and the related semiconductors $\mathrm{SnS}_{2}$, $\mathrm{SnSe}_{2}$ with respect to the vacuum level, for both monolayers and bulk, using density function theory (DFT). We then calculated band offsets of heterojunctions directly, using supercell models, to avoid specific models of band alignments. For the pure compounds, each supercell consists of the $\mathrm{MX}_{2}$ block and $20 \AA$ of vacuum layer. Six layers of $\mathrm{MX}_{2}$ are used to represent the bulk, based on the convergence of the band gap with layer number [24]. The electrostatic 
potential in the vacuum layer acts as the vacuum reference energy. For the direct calculations of band offsets, we use supercells containing 1 or 6 layers of TMD, representing the monolayer and bulk case. Often, the lateral lattice constants have a reasonable lattice match. For $(\mathrm{Sn}, \mathrm{Zr}, \mathrm{Hf}) \mathrm{X}_{2} /(\mathrm{Mo}, \mathrm{W}) \mathrm{X}_{2}$ heterojunctions, the mismatch is $\sim 15 \%$. Here, a $\sqrt{3} \times \sqrt{3}$ matching arrangement is used, with one TMD lattice rotated by $30^{\circ}$ with respect to the other, which then gives less than $3 \%$ lattice mismatch, Fig. 1. If the mismatch is small $(<4 \%)$, the lattices are strained laterally to an average of the two components. The $2 \%$ strain then gives a maximum $0.17 \mathrm{eV}$ energy error, based on previous calculations [10].

The calculations used the plane wave pseudopotential code CASTEP [25] with ultrasoft pseudo-potentials and plane wave cutoff of $400 \mathrm{eV}$, which converges total energies to $<0.01 \mathrm{eV}$ per atom. A $5 \times 5 \times 1 \mathrm{MP}$ k-point mesh is used for geometry optimization. The residual force is below $0.02 \mathrm{eV} / \mathrm{A}$. A $11 \mathrm{x} 11 \mathrm{x} 1 \mathrm{k}$-point mesh is used to calculate the density of states. Spin-orbit coupling is not included.

The lattice constants are relaxed with the PBE version of GGA. The Grimme [26] dispersion correction scheme corrects the DFT error of van der Waals bonding. The screened exchange (sX) hybrid functional [27] corrects the GGA band gap error. The amount of exact exchange and the screening length are kept fixed. This gives band gaps for the bulk in good agreement with experiment and GW [11]. For monolayers, the band gap is increased over the bulk value due to the absence of inter-layer dispersion. Additionally, the much lower screening creates large exciton binding energies [28], which further opens up quasi-particle band gaps [29-31]. Now, in real monolayer devices, the monolayers are not suspended, the gate dielectric and electrodes provide some screening, so the devices are not fully isolated. The screening is greater, the exciton binding energy reduces, and the band gap declines towards the optical gap $[29,30]$ as given by $\mathrm{sX}$.

Tables 1,2 give the calculated values of band gap, ionization potential and electron affinity within the sX functional, for both the bulk and monolayer cases, respectively. It also includes the calculated charge neutrality level (CNL) energy, a reference level for any metal induced gap states. The CNL energy here is referred to the valence band maximum. Experimental values of minimum band gap and ionisation potential are included for comparison.

Fig. 2 plots our calculated band edge energies with respect to the vacuum level to display chemical trends. For Mo and $\mathrm{W}$ compounds, our calculated values for monolayers are similar to those of Kang [7], who used the HSE functional. Our average of IP and EA energies for monolayers follows the average GW values of Rasmussen [10]. For bulk TMDs, our sX gaps are similar to the GW values of Jiang [11]. For monolayers, the calculated sX band gaps are less than the GW gaps [10] due to the large 2D exciton binding energy. We see that each series follows certain chemical trends. The EAs and IPs of the chalcogenide rise towards the vacuum level along the series sulfide, selenide and telluride, as their chalcogen $\mathrm{p}$ states rise towards the vacuum level. For the closed shell $\mathrm{d}^{0}$ systems $\mathrm{SnX}_{2}, \mathrm{ZrS}_{2}$ and $\mathrm{HfX}_{2}$, the valence band energies (IPs) are much deeper than for Mo and W systems, due to the filling of the additional $\mathrm{d}_{\mathrm{x} 2-\mathrm{y} 2} / \mathrm{d}_{\mathrm{xy}}{ }^{-}$ like valence band in these $\mathrm{d}^{2}$ systems. The band gaps open up going from bulk to monolayer, with roughly $80 \%$ of the increase occurring on the valence band side and $20 \%$ on the conduction band side. It is interesting to compare the calculated values with experiment. We see that our calculated IP values for bulk $\mathrm{MoS}_{2}, \mathrm{WSe}_{2}, \mathrm{WTe}_{2}, \mathrm{SnS}_{2}$ and $\mathrm{SnSe}_{2}$ are quite close to those measured by photoemission by Schlaf et al [32] and Kreis [33].

The conduction band offset (CBO) at a heterojunction can be expressed in terms of two limits $[20,34]$. In the electron affinity rule, the $\mathrm{CBO} \phi_{\mathrm{n}}$ is given as the difference between the two EAs,

$$
\phi_{\mathrm{n}}=\chi_{\mathrm{a}}-\chi_{\mathrm{b}} .
$$


In the strongly pinned limit, the $\mathrm{CBO}$ is given as the difference between the conduction band energies referred to the CNLs $\left(\Phi_{\mathrm{S}}\right)$ of semiconductor $\mathrm{a}$ or $\mathrm{b}$,

$$
\phi_{\mathrm{n}}=\left(\chi_{\mathrm{a}}-\Phi_{\mathrm{S}, \mathrm{a}}\right)-\left(\chi_{\mathrm{b}}-\Phi_{\mathrm{S}, \mathrm{b}}\right) .
$$

For the general case, the CBOs vary with a pinning factor $\mathrm{S}$ between the unpinned limit $(\mathrm{S}=1)$ of the EA rule, and the pinned limit $(S=0)$, as in Schottky barrier theory $[20,34,35]$,

$$
\phi_{\mathrm{n}}=\left(\chi_{\mathrm{a}}-\Phi_{\mathrm{S}, \mathrm{a}}\right)-\left(\chi_{\mathrm{b}}-\Phi_{\mathrm{S}, \mathrm{b}}\right)+\mathrm{S}\left(\Phi_{\mathrm{S}, \mathrm{a}}-\Phi_{\mathrm{S}, \mathrm{b}}\right)
$$

We calculated the band offsets for a number of critical heterojunctions by supercells and show the relevant partial density of states (PDOS) in Fig. 3. The VBOs for the limits $\mathrm{S}=0$ and $\mathrm{S}=1$ derived from the Tables 1,2 and eqn $(1,2)$, and the values calculated from supercells are compared in Table 3 and Fig 4. Figure 4 shows that the unpinned (EA) limit of S 1 describes well the offsets for stacked junctions. The valence band offsets vary over a wide range of $2.5 \mathrm{eV}$.

We now compare this with lateral heterojunctions. As there is lateral bonding, there must be lattice matching, as in 3D semiconductors. Various cases have been fabricated experimentally. The common anion case $\mathrm{MoS}_{2} / \mathrm{WS}_{2}$ is most common because of lattice matching. However, the more important case is for different anions, such as $\mathrm{MoS}_{2} / \mathrm{WSe}_{2}$, with a small $3.7 \%$ mismatch. This is handled in our supercell calculations by laterally straining to an average in-plane lattice constant. 10 cells of each component are used in the supercell. The effect of strain is allowed for using deformation potentials. Fig. 5 compares the PDOSs for $\mathrm{MoS}_{2} / \mathrm{WSe}_{2}$ heterojunctions for both the stacked and lateral cases, and the band offsets are summarised in Fig 5C. Clearly, there is a large difference in the $\mathrm{VBO}$ of $0.8 \mathrm{eV}$; the stacked case follows the unpinned $\mathrm{S}=1$ limit while the lateral case follows the pinned $S=0$ limit, showing almost no valence band offset. The big difference arises because $\mathrm{WSe}_{2}$ states lie higher than $\mathrm{MoS}_{2}$ states, but their CNLs both lie relatively close to their midgaps [37].

Now compare this with 3D semiconductors. In principle, there should be no difficulty deciding which model works. Yu [21] suggested that these heterostructures favored the CNL model. On the other hand, in a wide ranging calculation for tetrahedral semiconductors, Hinumi [22] found that the EA model worked reasonably well, but also found that the EA model and CNL models gave rather similar answers. Hoffling [23] studied band line-ups for semiconductors and oxides, and found both models were reasonable.

The problem is this; those semiconductors whose band edges lie well below the vacuum level (such as $\mathrm{ZnO}$ ) have CNLs which lies near or inside their conduction band. Semiconductors whose band edges lie high, close to the vacuum level, like $\mathrm{Cu}_{2} \mathrm{O}$ or $\mathrm{NiO}$, have CNLs that lie low in their gap [36]. Thus the electron affinities and CNLs follow the same chemical trends when comparing across a wide range of semiconductors, so that there are few practical cases to separate the two limits. We see from Fig 4 that the $\mathrm{MoS}_{2} / \mathrm{WSe}_{2}$ lateral heterojunction is a good test case to separate the cases, because each geometry must follow the different rules. Other pairs give the same result. We have checked the effect of strain on this conclusion.

Fig 5(d,e) compares alignments for the zigzag and armchair configurations for lateral $\mathrm{MoS}_{2} / \mathrm{WS}_{2}$ heterojunctions. There is little difference because the Mo-S bond is relatively nonpolar with only $0.3 e$ charge on each $\mathrm{S}$ site [47].

We now consider which heterojunctions are most useful for TFET applications. The simplest way to provide a narrow type II or type III alignment would be to use different anions, as in a $\mathrm{MoS}_{2} / \mathrm{MoTe}_{2}$ heterojunction. This would leave a net $0.6 \mathrm{eV}$ band gap between $\mathrm{MoTe}_{2} \mathrm{VB}$ and $\mathrm{MoS}_{2} \mathrm{CB}$ (Fig 2g). However, the poor stability of tellurides in $2 \mathrm{H}$ and $1 \mathrm{~T}$ phases argues against this choice. 
The other choice is to use different cations. Of particular interest are heterojunctions between $\mathrm{MoS}_{2}, \mathrm{MoSe}_{2}, \mathrm{WS}_{2}$ or $\mathrm{WSe}_{2}$ and $\mathrm{SnS}_{2}, \mathrm{HfS}_{2}$ or $\mathrm{ZrS}_{2}$. We noted above that the $\mathrm{d}^{0}$ semiconductors $\mathrm{SnS}_{2}, \mathrm{HfS}_{2}$ and $\mathrm{ZrS}_{2}$ have deeper lying VBMs, while the $\mathrm{d}^{2}$ semiconductors $\mathrm{MoX}_{2}$ and $\mathrm{WX}_{2}$ have higher lying VBMs due to the extra filled valence band. This can be used to give the desired narrow type II or type III band alignment. The key alignment is from the CBM of $\mathrm{SnS}_{2}$ to the VBM of the Mo or W chalcogenide. The VBMs rise along the series $\mathrm{S}, \mathrm{Se}$ and Te and the gap closes. The VBM of $\mathrm{SnS}_{2}$ is slightly deeper than for $\mathrm{HfS}_{2}$ or $\mathrm{ZrS}_{2}$. We see for heterojunctions with the Mo or W sulfide or selenide groups gives a type 2 or type 3 alignment, for the bulk or monolayer cases. For example, $\mathrm{MoS}_{2}$ or $\mathrm{WS}_{2}$ against $\mathrm{SnS}_{2}$ gives a type II alignment. On the other hand $\mathrm{SnS}_{2}$ against $\mathrm{MoSe}_{2}$ or $\mathrm{WSe}_{2}$ gives the desired type III offset, for both ML and bulk cases. When comparing the use of $\mathrm{SnS}_{2}, \mathrm{HfS}_{2}$ or $\mathrm{ZrS}_{2}$ as the counter layer, $\mathrm{HfS}_{2}$ or $\mathrm{ZrS}_{2}$ have greater thermodynamic stability [10], but $\mathrm{SnS}_{2}$ has a smaller $\mathrm{m}^{*}$ [10] and larger mobility. $\mathrm{SnS}_{2}$ and $\mathrm{SnSe}_{2}$ are disfavored for the CVD approach because $\mathrm{Sn}$ is polyvalent with both $\mathrm{Sn}^{2+}$ and $\mathrm{Sn}^{4+}$ states being possible.

Comparing with experiment, the calculated VBO for bulk $\mathrm{HfS}_{2}: \mathrm{WSe}_{2}$ heterojunctions $(1.9 \mathrm{eV})$ is close to that measured by photoemission by Tsipas [38] $(1.88 \mathrm{eV})$. Thus, the values for the bulk semiconductors are likely to be correct. On the other hand, the XPS value of VBO for $\mathrm{MoSe}_{2} / \mathrm{HfSe}_{2}$ heterojunctions $0.13 \mathrm{eV}$ is much less than our EA calculated value, $0.60 \mathrm{eV}$. The error is due to extrinsic effects in the CVD experiment, it was proposed that anion interstitial defects create a dipole layer which modifies the VBO [39]. This shows the need for careful control of chemical potential during growth.

We find that the much studied $\mathrm{MoS}_{2} / \mathrm{WSe}_{2}$ heterojunction gives a type II alignment. Recently, the VBO of monolayer $\mathrm{MoS}_{2} / \mathrm{WSe}_{2}$ heterojunction was measured by a combination of photoemission and scanning tunneling spectroscopy [40] to be $0.83 \mathrm{eV}$, which compares closely to our calculated value of $0.8 \mathrm{eV}$ in Table 3 .

Now, a number of groups have observed a notable interlayer photoluminescence in the $\mathrm{MoS}_{2} /$ $\mathrm{WSe}_{2}$ stacked heterojunction at $1.59 \mathrm{eV}$ [40-42]. This involves a direct transition between electrons in the $\mathrm{MoS}_{2} \mathrm{CB}$ to holes in the $\mathrm{WSe}_{2} \mathrm{VB}$, both at the $\mathrm{K}$ point [40] (Note as the CBs and VBs of each layer lie at different energies, the interlayer dispersion is less, by perturbation theory). A calculated PL energy from the sX band energies, assuming an EA band alignment, is $1.1 \mathrm{eV}$, much less than the observed value of $1.59 \mathrm{eV}$. Chiu [42] proposed to explain this using a VBO of $0.44 \mathrm{eV}$. However this contrasts with a measured photoemission VBO of $0.83 \mathrm{eV}$ [40], close to our calculated $0.8 \mathrm{eV}$. The problem can be resolved as follows, see Fig. 6; the isolated monolayers have very large exciton binding energies [29-31], but that the exciton binding energy declines quickly with the layer number, even for just two layers of the stacked system. The exciton energy is roughly $1.1 \mathrm{eV}$ in monolayer $\mathrm{MoS}_{2}$ or $\mathrm{WSe}_{2}$, but only $0.4 \mathrm{eV}$ in the bilayer [29,30]. Thus the observed PL energy can be rationalized according to the schematic of Fig. 6 .

In summary, we calculated the band alignments of various transition metal and non-transition metal dichalcogenides using supercells, for both stacked and lateral heterojunctions. The band offsets for stacked layer junctions followed the electron affinity rule whereas offsets for lateral heterojunctions follow the strongly-pinned limit, giving a key test for models of band alignments. The results for stacked-layer heterojunctions provided the best choices for use of TMDs in tunnel-FETs. The observed inter-layer photoluminescence energy was rationalized in terms of the band offsets and the strong change in the exciton binding energy.

We acknowledge funding by the EC project Grafol. 


\section{REFERENCES}

1. H Kroemer, Rev Mod Phys 73783 (2001)

2. A M Ionescu, H Riel, Nature 479329 (2011)

3. A Seabaugh, Q Zhang, Proc IEEE 982095 (2010)

4. S. Das, A. Prakash, R. Salazar, J. Appenzeller, ACS Nano 8, 1681 (2014).

5. D. Sarkar, X.J. Xie, W. Liu, W. Cao, J.H. Kang, Y.J.Gong, S. Kraemer, P. Ajayan, K. Banerjee, Nature 526, 91 (2015).

6. Y.C. Lin, et al, Nat Commun 6, 7311 (2015)

7. J. Kang, S. Tongay, J. Zhou, J.B. Li, J.Q. Wu, Appl Phys Lett 102, 012111 (2013).

8. Y.F. Liang, S.T. Huang, R. Soklaski, L. Yang, Appl Phys Lett 103042106 (2013).

9. G Gong, H.J. Zhang, W.H. Wang, L. Colombo, R.M. Wallace, K.J. Cho, Appl Phys Lett 103053513 (2013); (E) 107, 053513 (2015)

10. F.A. Rasmussen, K.S. Thygesen, J Phys Chem C 119, 13169 (2015)

11. H. Jiang, J Physl Chem $C$ 116, 7664 (2012).

12. $\quad$ M Y Li, et al, Science 349524 (2015)

13. X Duan et al, Nat Nano 91024 (2014)

14. C Zhang et al, Nat Comms 710349 (2016)

15. C Huang, et al, Nat Mats 131096 (2014)

16. Y.J. Gong, et al, Nat Mater 13, 1135-1142 (2014).

17. C G van der Walle, J Neugebauer, Nature 423626 (2003)

18. $\quad$ S H Wei, A Zunger, App Phys Lett 722011 (1998)

19. J. Tersoff, Phys Rev B 30, 4874 (1984)

20. J Robertson, J Vac Sci Technol B 18, 1785-1791 (2000).

21. E T Yu, J O McCaldin, T C McGill, Solid State Physics 461 (1992)

22. Y Hinuma, A Gruneis, G Kresse, F Oba, Phys Rev B 90155405 (2014)

23. B Hoffling, A Schliefe, C Rodl, F Bechstedt, Phys Rev B 85035305 (2012)

24. J E Padilha, H Peelaers, A Janotti, C G Van de Walle, Phys Rev B 90, 25420 (2014)

25. S. J. Clark, M.D. Segall, C.J. Pickard, P.J. Hasnip, M.J. Probert, K. Refson, M.C. Payne, Z Kristallogr 220, 567 (2005).

26. S. Grimme, J Comput Chem, 27, 1787 (2006).

27. S.J. Clark, J. Robertson, Phys Rev B 82, 085208 (2010).

28. A.T. Hanbicki, M. Currie, G.Kioseoglou, A.L. Friedman, B.T. Jonker, Solid State Commun 203, 16 (2015).

29. T. Cheiwchanchamnangij, W.R.L. Lambrecht, Phys Rev B 85205302 (2012).

30. H.P. Komsa, A. Krasheninnikov, Phys Rev B 86241201 (2012).

31. A. Ramasubramaniam, Phys Rev B 86, 115409 (2012)

32. R. Schlaf, O. Lang, C. Pettenkofer, W. Jaegermann, J Appl Phys 85, 2732 (1999).

33. C. Kreis, M. Traving, R.Adelung, L. Kipp, M. Skibowski, Appl Surf Sci 166, 17 (2000).

34. W. Monch, Phys Rev Lett 58, 1260 (1986).

35. J. Tersoff, Phys Rev Lett 52465 (1984)

36. J Robertson, S J Clark, Phys Rev B 83075205 (2011)

37. Y Guo, D Liu, J Robertson, App Phys Lett 106173106 (2015)

38. P Tsipas, D Tsoutsou, J Marquez-Velasco, K E Aretouli, E Xenogiannopoulou, E Vassalou, G Kordas, A. Dimoulas, Microelectron Eng 147, 269-272 (2015)

39. K.E. Aretouli, P. Tsipas, D. Tsoutsou, J. Marquez-Velasco, E Xenogiannopoulou, S.A. Giamini, E. Vassalou, N Kelaidis, A Dimoulas, Appl Phys Lett 106143105 (2015)

40. M.H. Chiu, C.D. Zhang, H.W. Shiu, C,P. Chuu, C.H. Chen, C.Y.S. Chang, C.H. Chen, M.Y.Chou, C.K. Shih, L.J. Li, Nat Commun 106, 143105 (2015) 
41. H. Fang, C. Battaglia, C. Carraro, S. Nemsak, B. Ozdol, J.S. Kang, H.A. Bechtel, S.B.Desai, F. Kronast, A.A. Unal, G.Conti, C. Conlon, G.K. Palsson, M.C. Martin, A.M. Minor, C.S. Fadley, E. Yablonovitch, R. Maboudian, A. Javey, Natl Acad Sci USA 111, 6198 (2014)

42. M.H. Chiu, M.Y. Li, W.J. Zhang, W.T. Hsu, W.H. Chang, M. Terrones, H. Terrones, L.J. Li, ACS Nano 8, 9649 (2014)

43. R Cheng, D H Li, H L Zhou, C Wang, A X Yin, S Jiang, Y Liu, Y Chen, Y Huang, X F Duan, Nano Lett 14, 5590 (2014)

44. G Gong, L Colombo, RM Wallace, K Cho, Nano Lett. 14, 1714 (2014)

45. J Kang, W Liu, D Sarkar, D. Jena, K Banerjee, Phys. Rev. X 4, 031005 (2014)

46. Y Guo, D Liu, J Robertson, ACS Appl Mater Interfaces 7, 25709 (2015)

47. C Ruppert, O B Aslan, T F Heinz, Nanoletts 146231 (2014)

48. $\quad$ D L Greenaway, R Ntische, J Phys Chem Solids 261445 (1965)

49. K Terashima, I Imai, Solid State Comms 63315 (1987) 


\section{TABLES.}

\begin{tabular}{|l|l|l|l|l|l|l|}
\hline $\begin{array}{l}\text { Bulk / } \\
\text { eV }\end{array}$ & Gap & $\begin{array}{l}\text { Gap } \\
\text { (exp) }\end{array}$ & IP & EA & CNL & $\begin{array}{l}\text { Exp. } \\
\text { IP }\end{array}$ \\
\hline $\mathrm{MoS}_{2}$ & 1.35 & 1.29 & 5.56 & 4.21 & 0.7 & 5.47 \\
\hline $\mathrm{MoSe}_{2}$ & 1.16 & 1.09 & 4.97 & 3.81 & 0.43 & \\
\hline $\mathrm{MoTe}_{2}$ & 1.01 & 0.93 & 4.79 & 3.78 & 0.33 & \\
\hline $\mathrm{WS}_{2}$ & 1.44 & 1.57 & 5.36 & 3.92 & 0.88 & \\
\hline $\mathrm{WSe}_{2}$ & 1.33 & 1.2 & 4.83 & 3.50 & 0.77 & 5.19 \\
\hline $\mathrm{WTe}_{2}$ & 1.07 & & 4.57 & 3.5 & 0.39 & \\
\hline $\mathrm{HfS}_{2}$ & 1.95 & 1.98 & 6.70 & 4.75 & 0.95 & 7.10 \\
\hline $\mathrm{HfSe}_{2}$ & 1.25 & 1.14 & 5.90 & 4.65 & 0.6 & \\
\hline $\mathrm{ZrS}_{2}$ & 1.8 & 1.78 & 6.75 & 4.95 & 0.85 & \\
\hline $\mathrm{ZrSe}_{2}$ & 1.12 & 1.18 & 5.97 & 4.85 & 0.56 & \\
\hline $\mathrm{SnS}_{2}$ & 2.0 & 2.18 & 7.3 & 5.3 & 1.33 & 7.14 \\
\hline $\mathrm{SnSe}_{2}$ & 1.3 & 1.03 & 6.3 & 5.0 & 0.87 & 6.21 \\
\hline
\end{tabular}

Table 1. Band gap, experimental optical band gap [47-49], ionization potential (IP, VB energy), electron affinity (EA, CB energy), and charge neutrality level of bulk TMDs calculated in the sX functional for bulk TMDs. The ionisation potential is compared to experimentally IPs from photoemission [32].

\begin{tabular}{|l|l|l|l|l|l|}
\hline $\begin{array}{l}\text { ML / } \\
\text { eV }\end{array}$ & Gap & $\begin{array}{l}\text { Gap } \\
\text { (exp) }\end{array}$ & IP & EA & CNL \\
\hline $\mathrm{MoS}_{2}$ & 1.88 & 1.88 & 5.98 & 4.1 & 0.95 \\
\hline $\mathrm{MoSe}_{2}$ & 1.71 & 1.55 & 5.41 & 3.70 & 0.84 \\
\hline $\mathrm{MoTe}_{2}$ & 1.46 & 1.1 & 5.11 & 3.65 & 0.72 \\
\hline $\mathrm{WS}_{2}$ & 2.13 & 1.99 & 5.88 & 3.75 & 1.07 \\
\hline $\mathrm{WSe}_{2}$ & 1.82 & 1.63 & 5.17 & 3.35 & 0.73 \\
\hline $\mathrm{WTe}_{2}$ & 1.31 & & 4.76 & 3.45 & 0.6 \\
\hline $\mathrm{HfS}_{2}$ & 2.38 & & 7.03 & 4.65 & 1.29 \\
\hline $\mathrm{HfSe}_{2}$ & 1.48 & & 6.07 & 4.59 & 0.73 \\
\hline $\mathrm{ZrS}_{2}$ & 2.0 & & 6.9 & 4.9 & 1.06 \\
\hline $\mathrm{ZrSe}_{2}$ & 1.40 & & 6.19 & 4.79 & 0.78 \\
\hline $\mathrm{SnS}_{2}$ & 2.4 & & 7.6 & 5.2 & 1.55 \\
\hline $\mathrm{SnSe}_{2}$ & 1.6 & & 6.54 & 4.94 & 1.10 \\
\hline
\end{tabular}

Table 2. Band gap, experimental optical band gap, ionization potential (VB energy), electron affinity (CB energy), and charge neutrality level of a monolayer TMDs calculated in the sX functional. 


\begin{tabular}{|l|l|l|l|}
\hline Monolayers (eV) & $\mathrm{S}=1$ & $\mathrm{~S}=0$ & supercell \\
\hline $\mathrm{MoS}_{2} / \mathrm{SnS}_{2}$ & 1.56 & 0.6 & 1.9 \\
\hline $\mathrm{MoSe}_{2} / \mathrm{SnS}_{2}$ & 2.33 & 0.71 & 2.5 \\
\hline $\mathrm{MoTe}_{2} / \mathrm{SnS}_{2}$ & 2.43 & 0.83 & 2.5 \\
\hline $\mathrm{MoS}_{2} / \mathrm{HfS}_{2}$ & 1.1 & 0.34 & 1.5 \\
\hline $\mathrm{MoSe}_{2} / \mathrm{HfS}_{2}$ & 1.67 & 0.45 & 1.9 \\
\hline $\mathrm{WSe}_{2} / \mathrm{SnS}_{2}$ & 2.37 & 0.82 & 2.6 \\
\hline $\mathrm{WSe}_{2} / \mathrm{HfS}_{2}$ & 1.91 & 0.56 & 1.8 \\
\hline $\mathrm{WS}_{2} / \mathrm{HfS}_{2}$ & 1.2 & & 1.8 \\
\hline $\mathrm{MoS}_{2} / \mathrm{WSe}_{2}$ & 0.81 & 0.22 & 0.75 \\
\hline $\begin{array}{l}\text { lateral } \mathrm{MoS}_{2} / \\
\mathrm{WSe}_{2}\end{array}$ & 0.22 & 0 \\
\hline
\end{tabular}

Table 3. Calculated valence band offsets $(\mathrm{eV})$ for stacked monolayer heterojunctions of the semiconductor pairs indicated, from the electron affinity rule $(S=1)$, matched charge neutrality levels $(S=0)$, and by direct calculation in supercells. Final row is for lateral heterojunction. 
Figure Captions

1. Top view of a stacked supercell of on $\mathrm{MoS}_{2}$ on $\mathrm{SnS}_{2}$ with the $\mathrm{SnS}_{2}$ lattice rotated by $30^{0}$ in the $\sqrt{3} \times \sqrt{3}$ arrangement. Orange balls are $S$ atoms belonging to the lower $\operatorname{SnS}_{2}$.

2. Chemical trends of electron affinities and ionization potentials of various transition metal dichalcogenides.

3. Calculated band offsets for (a) $\mathrm{ML}$ and (b) bulk $\mathrm{MoS}_{2} / \mathrm{SnS}_{2}$ stacked heterojunctions. Calculated band offsets for (c) $\mathrm{ML}$ and (d) bulk $\mathrm{HfS}_{2} / \mathrm{WSe}_{2}$ stacked heterojunctions. Calculated band offsets for (e) $\mathrm{ML}$ and (f) bulk $\mathrm{SnS}_{2} / \mathrm{WSe}_{2}$ stacked heterojunctions.

4. Calculated VBOs from the supercell method plotted against VBOs expected from the electron affinity rule, from Table 2. Also shown are the expected VBOs in strongly pinned limit of aligned CNLs.

5. (a) Calculated partial densities of states for $\mathrm{ML} \mathrm{MoS}_{2} / \mathrm{WSe}_{2}$ for (a) stacked layers and (b) lateral junction. (c) summary comparison on band alignments for stacked and lateral junctions. (d,e) $\mathrm{MoS}_{2} / \mathrm{WS}_{2}$ lateral heterojunctions, for zigzag and armchair configurations. The small difference reflects the relatively non- polar bonding in $\mathrm{MoS}_{2}$.

6. Schematic band alignment at a stacked heterojunction of $\mathrm{ML} \mathrm{MoS}$ and $\mathrm{WSe}_{2}$, showing the intralayer PL and the interlayer (IL) PL, and how the reduction of exciton binding energy for a 2-layer system can rationalize the observed $1.59 \mathrm{eV}$ luminescence. An exciton binding energy of $1.1 \mathrm{eV}$ is used for $\mathrm{ML} \mathrm{MoS}, 0.87 \mathrm{eV}$ for $\mathrm{ML} \mathrm{WSe}$, and $0.4 \mathrm{eV}$ for the $\mathrm{MoS}_{2} / \mathrm{WSe}_{2}$ bilayer. 


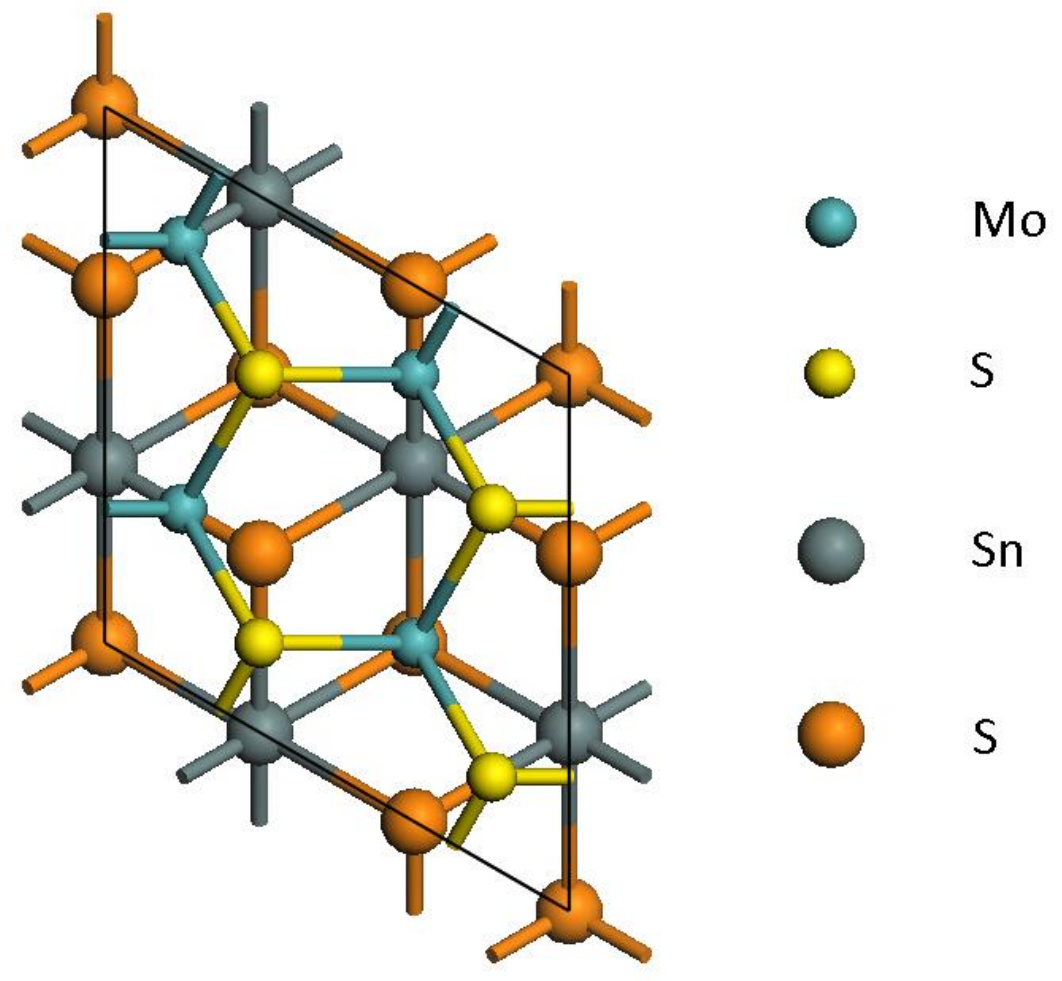

Fig. 1. 


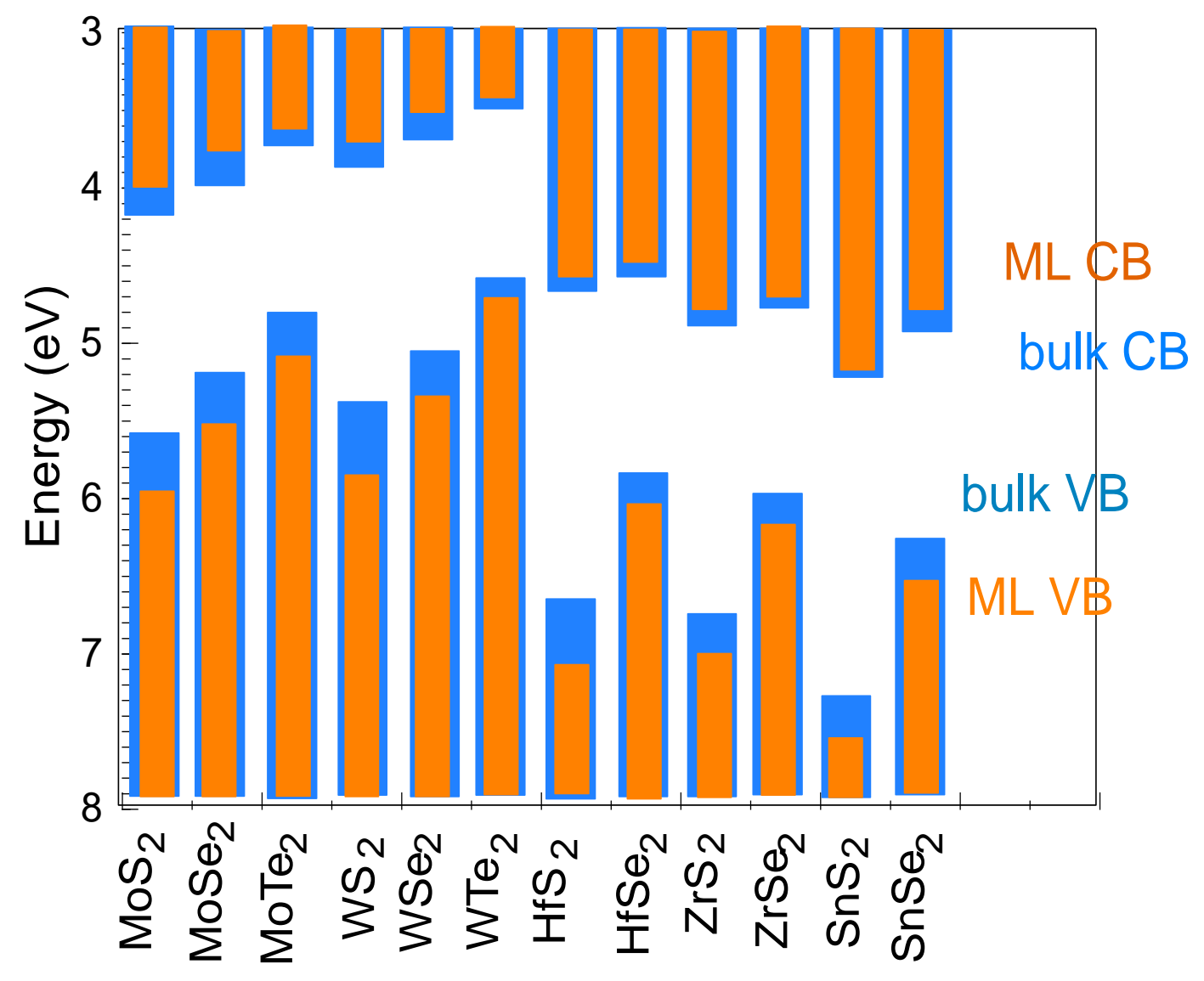

Fig 2 
a

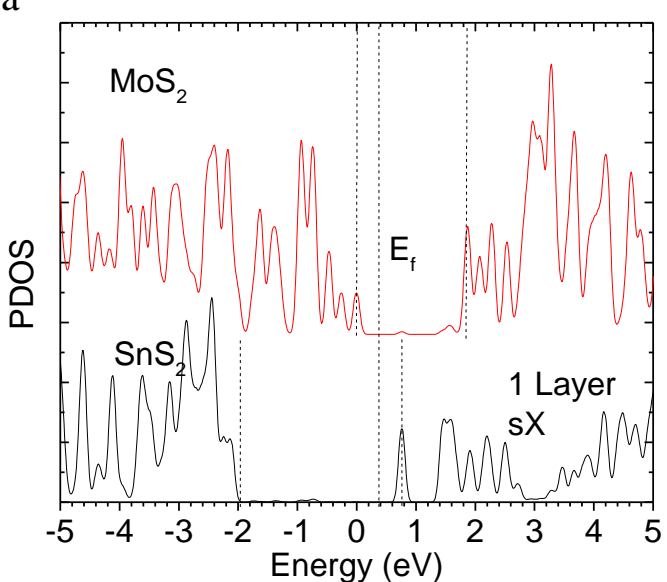

c

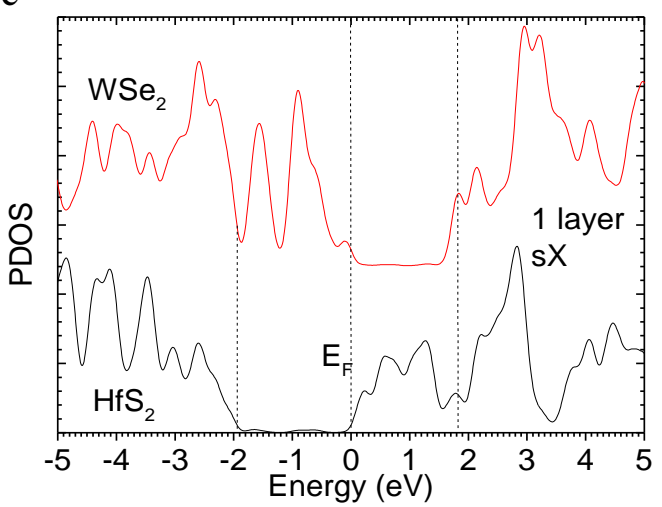

e

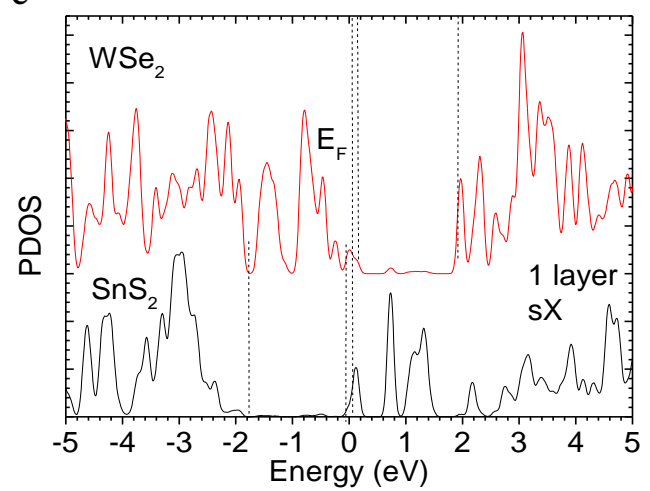

g

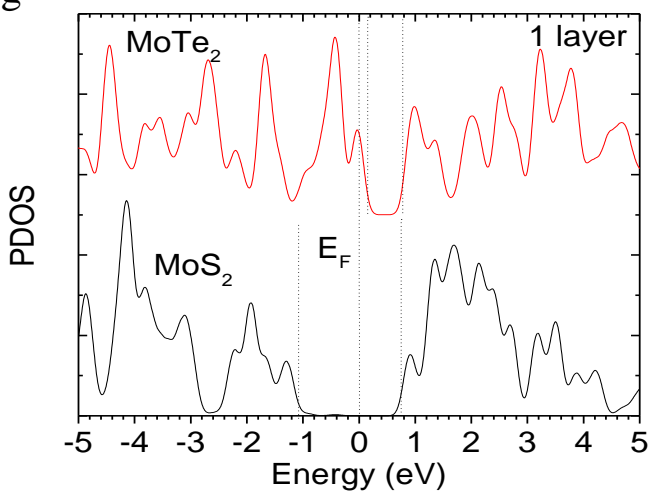

b

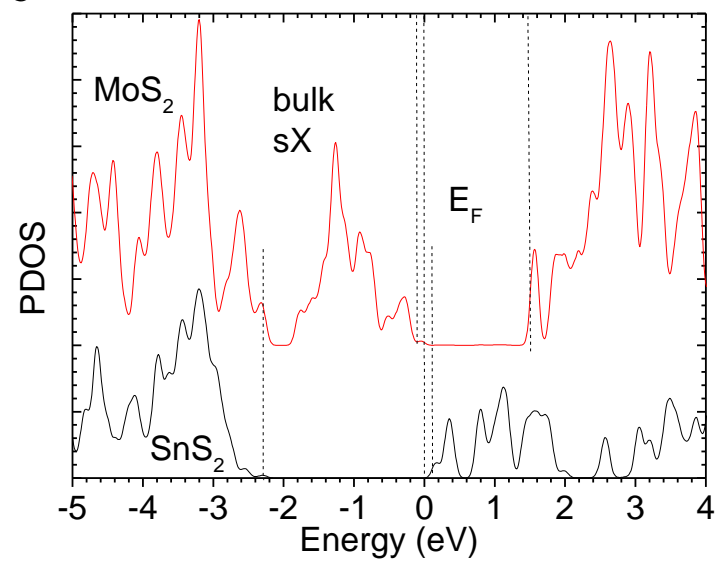

d

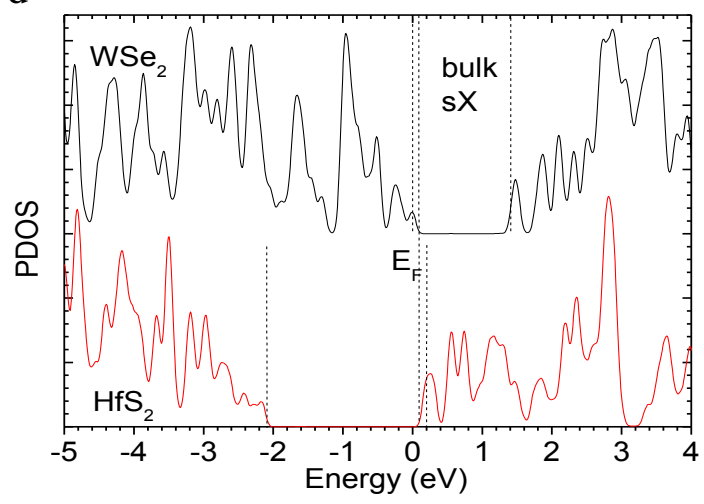

f

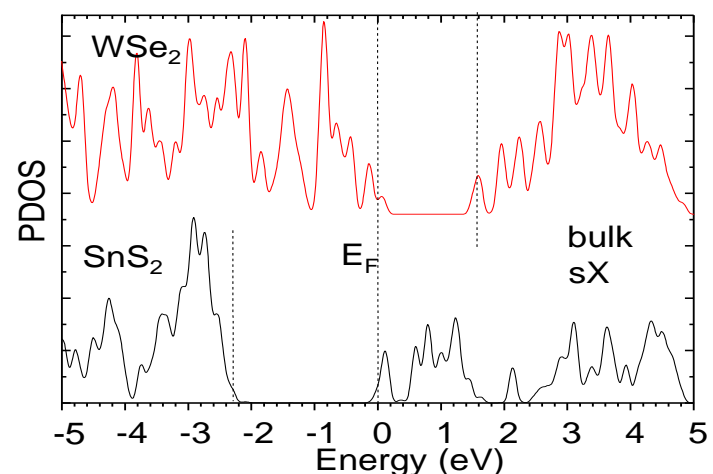

Fig 3 


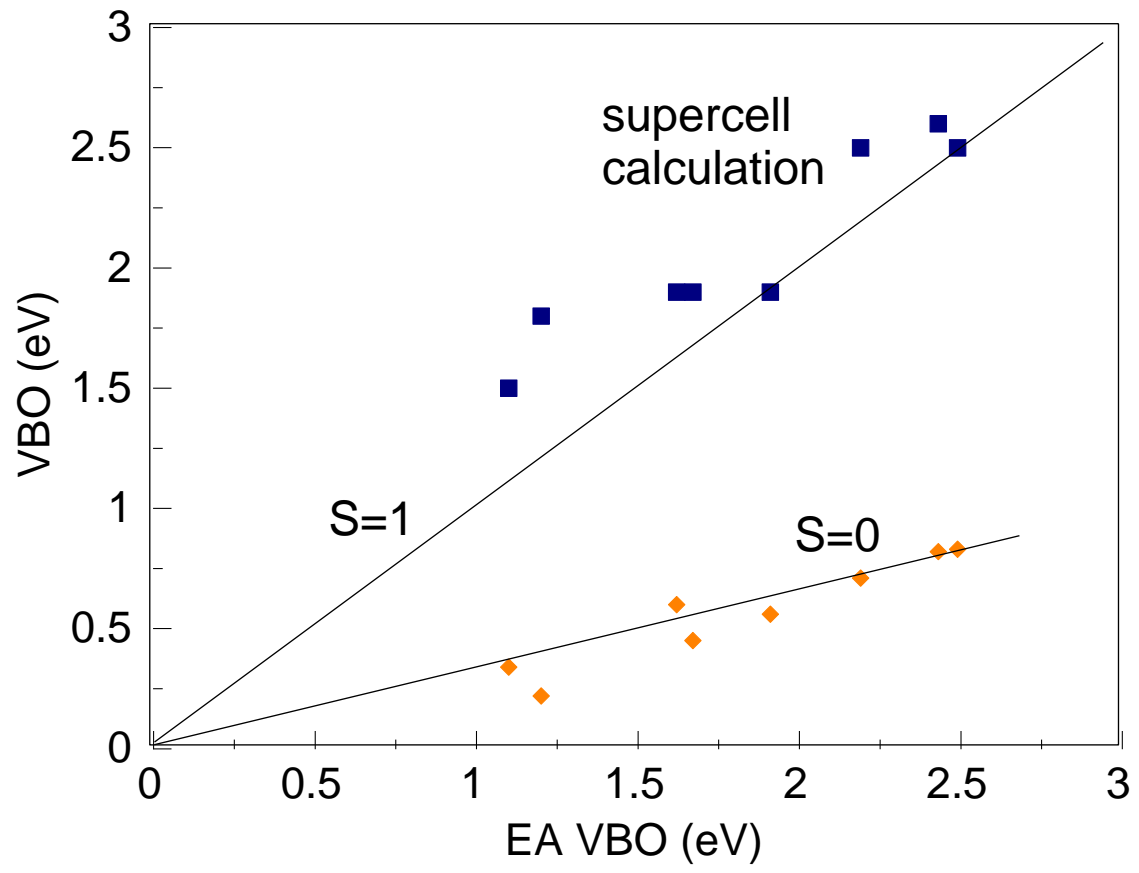

Fig. 4 
a

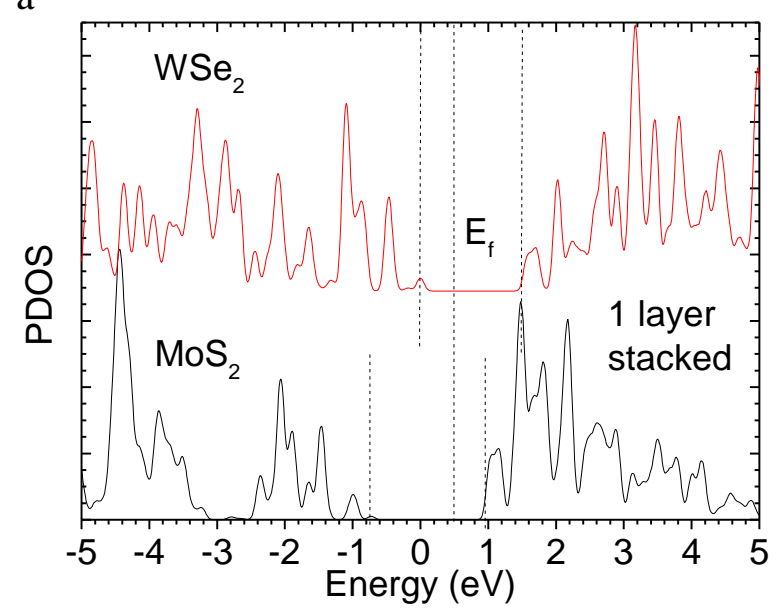

c

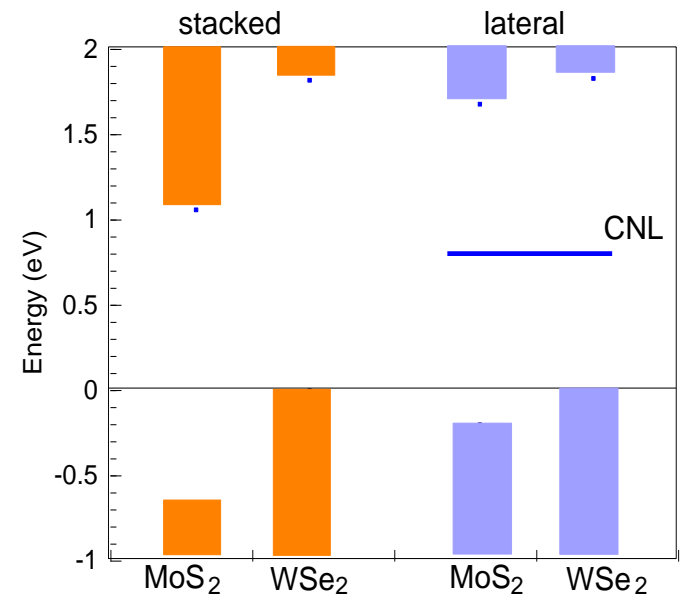

d

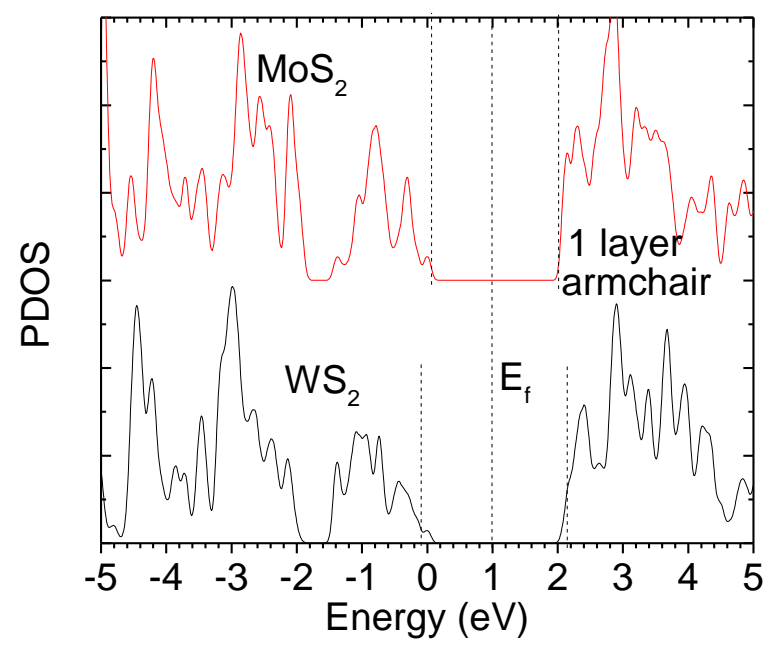

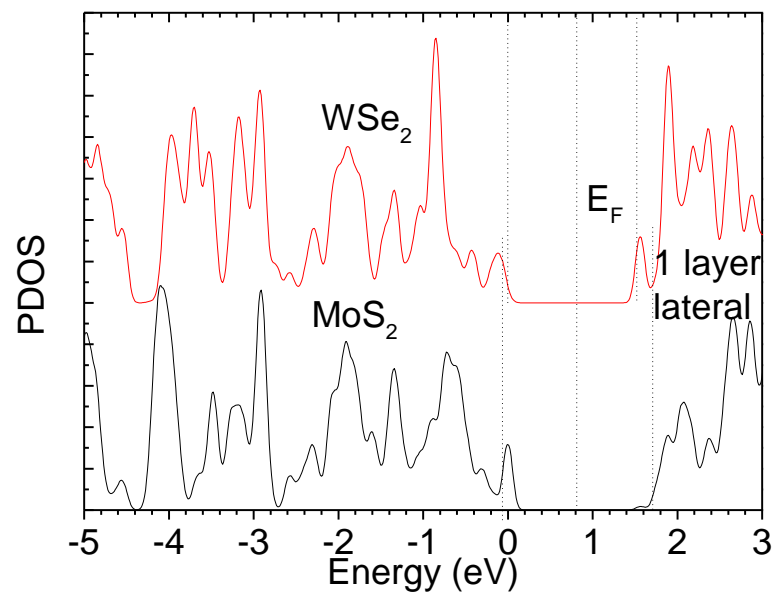

e

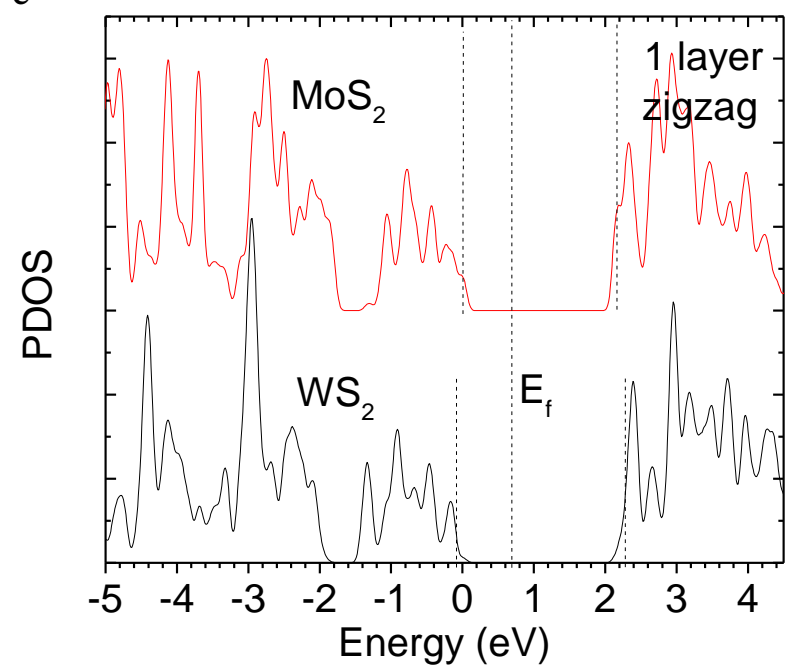

Fig. 5 


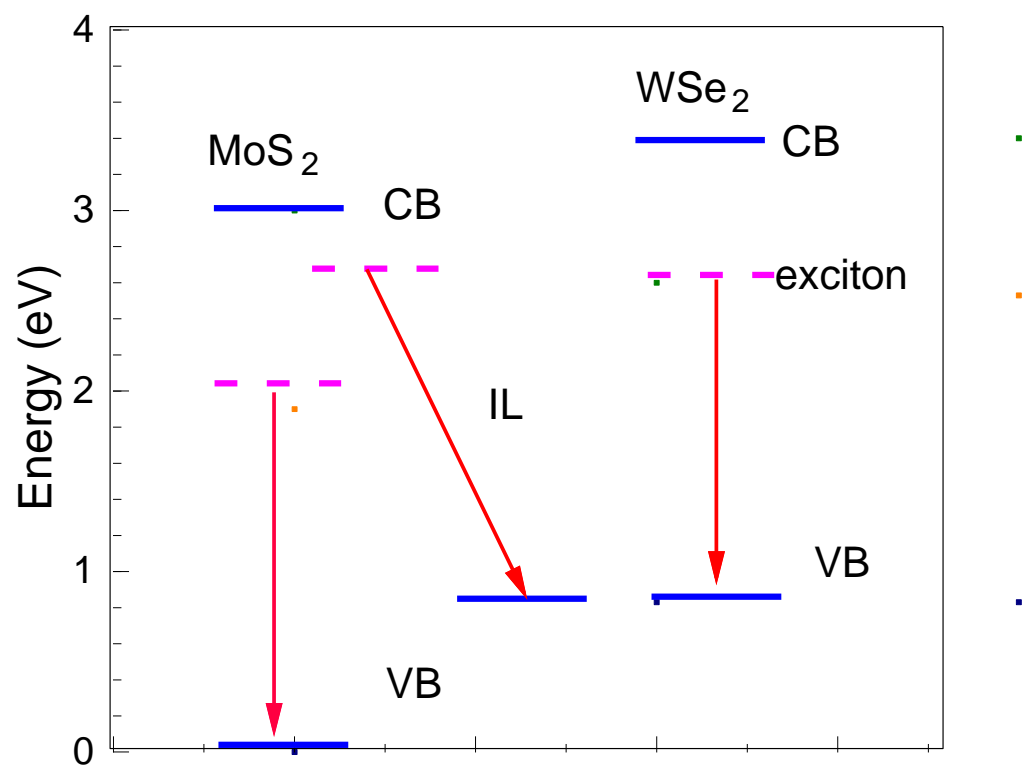

Fig 6 